\title{
A Modal Approach to the Modeling of Rectangular Detector Horns and Cavities at $\mathrm{THz}$ Frequencies
}

\author{
Stephen Doherty, Neil Trappe, Créidhe O'Sullivan, Anthony Murphy \\ Dept. of Experimental Physics, NUI-Maynooth, Ireland
}

Far-IR space telescopes use horn antenna coupled bolometers for achieving high levels of sensitivity. Unfortunately at $\mathrm{THz}$ frequencies large cavity coupled horns prove significantly challenging to simulate accurately. This paper will outline alternative simulation concepts for such problems.

\section{INTRODUCTION AND BACKGROUND}

$\mathrm{T}_{\mathrm{s}}^{\mathrm{h}}$ he modal approach or mode matching technique to simulate single moded cylindrical horns has been successfully employed several times in the past and shows excellent agreement with both measurements and alternative modeling techniques. In this procedure described in detail in [1] the basis set of waveguide TE and TM modes are represented by Bessel functions. This results in relatively efficient expressions to calculate a full $\mathrm{S}$ parameter description of the horn with aperture or farfield pattern predictions. This technique can be expanded to model rectangular or pyramidal horns and here the basis set of modes are expressed as a linear expression of sinus and cosine terms.

In comparison to other electromagnetic simulation techniques this approach proves advantageous when considering simple geometric structures, which are modeled by propagating modes and conserving power at sub wavelength sections that when cascaded together represent a full detector horn. For such structures a modal analysis requires significantly less computational power and time when compared with more generic FIT modeling techniques.

In this paper we describe the approach to modeling large horns for far infrared astronomy applications including the horn antenna coupled cavity housing a Transition Edge Sensors (TES) detector, proposed for the SAFARI instrument of the joint JAXA/ESA SPICA mission. It is difficult to model the horn antenna integrated with the cavity and a few different analysis approaches are explored here.

Although the geometry of these cavity regions preclude them from being considered solely by such modal techniques, it is proposed that a combination of both the modal and a Method of Moments approach could successfully simulate the entire system.

\section{RESULTS}

Excellent agreement has been obtained between the mode matching code developed at NUI-Maynooth using modal analysis and independently run CST simulations utilising finite integration techniques for a number of simpler examples already. A sample of this agreement can be seen in the following figure, which displays the simulated far-fields of a single moded cylindrical horn at $661 \mathrm{GHz}$.

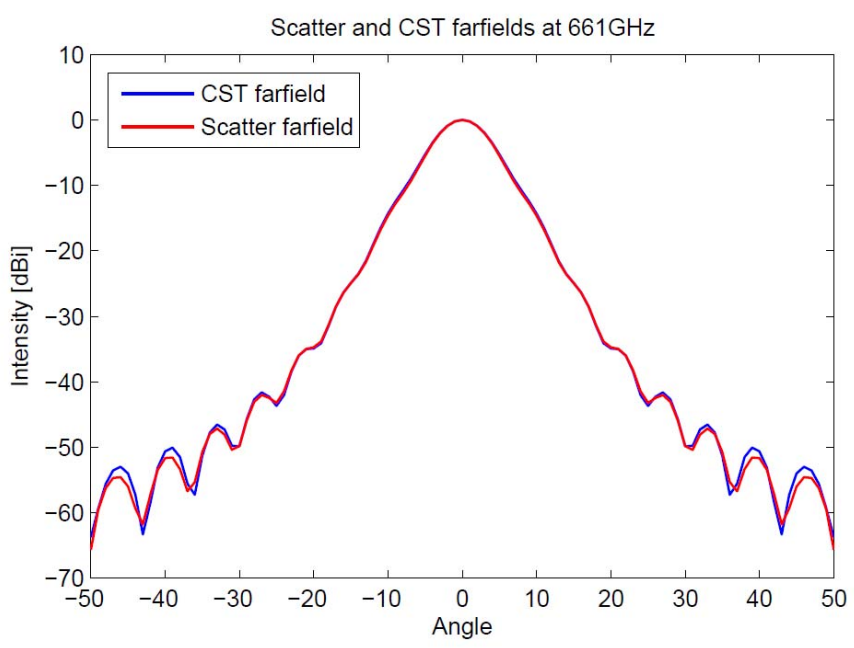

Computational restraints prevent CST from being used to simulate the detector cavities integrated with the antenna of interest for the SAFARI instrument due to geometric size. A more general examination of detector cavities is being carried out with the intent of eventually achieving the development of code capable of modeling electrically large detector cavities coupled to a horn antenna.

\section{REFERENCES}

[1] J.A. Murphy and A. Egan, "Examples of Fresnel diffraction using Gaussian modes," in Eur. J. Phys. 14, 1993, pp. 121-127. 